\title{
Assessing the Knowledge, Attitude and Practice of Menstrual Hygiene Management Among Junior High Schools Adolescent Females in the Yendi Municipality in the Northern Region of Ghana
}

\author{
Akwasi Boakye-Yiadom \\ David Alatule Aladago \\ University for Development Studies, School of Allied Health Sciences, \\ Department of Public Health, Tamale \\ Julien Beweleyir \\ Hamza Bawa Mohammed \\ Marian Fairuza Salifu \\ University for Development Studies, School of Allied Health Sciences, \\ Department of Nursing, Tamale \\ Asaarik, Mathias \\ Tamale Teaching Hospital-Department of Public Health, Tamale
}

Doi: 10.19044/esj.2018.v14n36p467 URL:http://dx.doi.org/10.19044/esj.2018.v14n36p467

\begin{abstract}
Introduction: Every year, different batches of adolescent girls between the ages of 10-19 years experience menarche often unprepared to manage the menstruation hygienically. In Ghana, most girls enter adolescence when they are in basic schools, which make menstrual hygiene among girls in Junior High Schools very important. This study examined the knowledge, attitudes, and practices of menstrual hygiene management among both premenarcheal and post-menarcheal adolescent students in basic schools in the Yendi Municipality in Ghana. Methods: the study was cross sectional and used quantitative research methods. Semi-structured questionnaires were used to collect data from the female adolescent students aged 10-19 years. A total of 412 female adolescents from 9 basic schools in the Yendi Municipality participated in the study. Students were scored for their level of knowledge, attitude and practices of menstrual hygiene management. Results: The study found that although awareness of menstruation was universal, the Likert scores showed that $67.5 \%$ had adequate knowledge of menstrual hygiene management. About a tenth (13.6\%) of the adolescent female students had a positive attitude towards menstrual hygiene management and a third (31.1\%) practised good menstrual hygiene management. Older age and living with both
\end{abstract}


parents were the main predictors of knowledge of menstrual hygiene management $(\mathrm{P}<0.05)$. Similarly, age, staying with both parents, staying with mother only, access to funds and having adequate knowledge of menstruation were the main determinants of good menstrual hygiene management practice among adolescent girls in basic schools in Yendi Municipality $(\mathrm{P}<0.05)$. Conclusions and recommendations: more than half of the respondents have adequate knowledge but their awareness and knowledge did not translate to positive attitude towards good menstrual hygiene management practices due to negative socio-cultural norms and practices among others that tend to stigmatise menstruation.

Keywords: Adolescents, Menstruation, Menstrual hygiene management, Knowledge, Attitude

\section{Introduction}

Different batches of adolescents (people between the ages of 10-19 years) enter puberty annually; unaware, unprepared and confused by the physical changes and emotional challenges that come with it. Hygienically managing menstruation in adolescence can be challenging and enigmatic. Social norms and cultural practices surrounding menstruation means that most adolescents receive incomplete or inaccurate information on menstrual hygiene management (MHM). The lack of practical life skills to effectively manage menstruation could reduce adolescent girls' self-esteem, impair their health and truncate their education. The United Nations Children's Fund (UNICEF) has been at the forefront of efforts to provide menstruation education; and water, sanitation and hygiene (WASH) facilities in schools. Despite the critical importance of MHM to adolescent girls, studies show that most educational institutions do not provide adolescents with the necessary information prior to menarche. Mothers and female family members, who may not have the necessary knowledge and skills in MHM, are often the main source of information for most adolescents (Chandra-Mouli \& Patel, 2017). MHM means "Women and adolescent girls use a clean material to absorb or collect menstrual blood, and this material can be changed in privacy as often as necessary for the duration of the menstrual period" (Sommer et al., 2017). It also includes the use of soap and water for washing the body as often as necessary and access to the necessary facilities for the disposal of used menstrual management materials. Literature shows that most adolescents have inaccurate knowledge and misconceptions about menstruation. Those that have knowledge tend to practice wrong menstrual hygiene management due to factors such as inadequate menstrual sanitary materials and lack of emotional or physical support (Chandra-Mouli \& Patel, 2017). 
In a study in (Yadav et al., 2017) found that although 67.4\% of female adolescent students were aware of MHM, about a quarter (26.4\%) of them had adequate knowledge of MHM. In Western Ethiopia, (Upashe et al., 2015) found that $60.9 \%$ of them had adequate knowledge of MHM. They also discovered that teachers were their primary source of MHM information. However, in Nepal, (Gultie et al., 2014) revealed that majority (90.7\%) of adolescent female students had high level of knowledge on MHM. (Gultie et al., 2014) also found that place of residence and maternal educational status were predictors of knowledge about MHM. Although menstruation is a normal biological process and a key sign of reproductive health, ignorance and misconceptions often lead to shaming and marginalisation of menstruating girls (Santra, 2017). A systematic review revealed that less than half of adolescents in India have menstrual information prior to menarche (Van Eijk et al., 2016). Among adolescent students in Katsina state of Nigeria, (OkaforTerver \& Chuemchit, 2017) discovered that although about a third of them had basic knowledge of menstruation, 3-in-5 had no knowledge of the cause of menses, the channel through which menses flow and intervals between menstruation. They also found that knowledge was a predictor of proper menstrual hygiene practice. In Nepal, (Ghimire, 2017) found that among adolescent girls, 5\% had good knowledge of MHM. (Upashe et al., 2015) found a statistically significant association between educational status mothers and adequate knowledge of MHM among student adolescent girls.

Regarding attitude towards menstruation, studies show that about half of adolescent girls have a positive attitude towards menstrual hygiene management (Yadav et al., 2017). In India for example, menstruating adolescent girls experience religious restrictions with a quarter (24\%) of them missing school during periods (Van Eijk et al., 2016). In Uganda, findings by (Miiro et al., 2018) suggest nearly a fifth of adolescent female students missed school due to pains associated with menstruation. Additionally, menstruation is considered a curse, disease or sin by adolescent girls in Uganda (Boosey et al., 2014). Prior to receiving health education at school, $72.4 \%$ of girls in India considered menstrual blood impure ( Nemade et al., 2009). Adolescent girls also refrained from household work including cooking or performing religious activities during menstruation. In developing countries, absenteeism in schools due to menstruation range from $2 \%$ of urban-adolescents in Nigeria to $61.7 \%$ of their rural counterparts in Uganda (Chandra-Mouli \& Patel, 2017). Early menarche is associated with early sexual initiation, early pregnancy, early marriage and some sexually transmitted infections among adolescent girls in low and middle-income countries (Ibitoye et al., 2017). Among adolescent female students in Bangladesh, discomfort at school during menstruation, restriction from performing any activity during menstruation 
and perception that menstrual problems interfered with school performance were associated with absenteeism (Alam et al., 2017).

Moreover, (Yadav et al., 2017) found that less than half (40\%) of adolescent girls practised good menstrual hygiene. Unlike rural areas where about a third of adolescent girls use commercial sanitary pads, the majority of urban adolescent girls use sanitary pads during menstruation (Van Eijk et al., 2016; Barthakur \& Barkataki, 2017) found that a third of girls changed their absorbents in school facilities but, approximately a quarter of adolescent girls dispose of their used menstrual materials inappropriately. (Hennegan et al., 2016) discovered that female adolescent students in Uganda prefer reusable pads. However, in areas where there are no support structures, menstruating girls may withdraw, resort to unhygienic practices or miss school for the duration of menstruation. Some of them use improvised materials including cotton wool, cloth, old pieces of mattresses, newspapers and leaves as absorbents (Chandra-Mouli \& Patel, 2017; Trinies et al., 2015; Kuhlmann et al., 2017). (Chandra-Mouli \& Patel, 2017) also noted that a little over a fifth of girls in India and Egypt changed their absorbents in school compared to about half of their counterparts in Nigeria. A study in one urban area in India found that over 9-in-10 adolescent girls said they had access to water and a toilet facility in schools (Gultie et al., 2014). Studies suggest that urban adolescents bath more often than their rural colleagues during menstruation. Studies also show that most adolescents in India clean their genitalia at least twice a day during menstruation. Genital cleaning is also associated with prior MHM education (Chandra-Mouli \& Patel, 2017). Although a systematic review found no significant association between sanitary pad provision and school attendance, a randomized control trial (RCT) in Ghana suggested that MHM education together with provision of free pads could improve school attendance among adolescent girls (Montgomery et al., 2012; Hennegan et al., 2016). (Upashe et al., 2015) found that access to funds was a predictor of good practice of MHM.

Despite the apparent dearth of literature on MHM among adolescent female students in Ghana, studies elsewhere suggest that most adolescent girls lack knowledge of proper MHM (Chandra-Mouli \& Patel, 2017). Most postmenarcheal adolescents are unable to maintain their dignity during menstruation due to lack of access to safe and hygienic absorbents for management of menstrual blood. They may miss several school days every month or drop out of school altogether due to menstruation. Post-pubescent adolescent females also stand the risk of unintended pregnancy if they are not adequately educated on the changes to their reproductive system and safe sex. UNICEF recommends that MHM education begins in primary schools so that girls understand the changes in their bodies and reproductive health system prior to menarche (Dutta, 2016). However, in most developing countries, both 
parents and schools do not seem to prepare adolescent girls enough for menstrual hygiene management prior to the onset of menarche. According to the Ghana Demographic and Health Survey (GDHS, 2014), adolescents constitute $22 \%$ of Ghana's population, with girls forming the majority. The Yendi Municipal in northern Ghana has at least 2,711 adolescent girls in Junior High Schools (JHSs) (GSS, 2014). However, there is no study of MHM among adolescent female students in the Yendi Municipality. This study, thus, assessed the knowledge, attitude and practices of MHM among adolescent girls aged 10-19 years in basic schools in the Yendi Municipality of Northern Region, Ghana.

\section{Methodology}

This is a cross-sectional descriptive quantitative study. Simple random sampling without replacement technique was used to select the participants. A list of JHSs in the Yendi Municipality was obtained from the Ghana Education Service (GES). There are $37 \mathrm{JHSs}$ located in nine circuits, with a total of 3,503 female students. One school was randomly picked from each of the nine circuits. All adolescent girls between the ages of 10-19 who attended the selected schools on the interview day were included in the study. A total of 430 adolescent girls participated in the study. Semi-structured questionnaires were used to collect information from the adolescent girls. The data were entered and cleaned using Ms Excel and analysed using IBM SPSS Statistics software version 23 .

To assess the level of knowledge of menstrual hygiene management eight questions were posed and every correct answer was given a score of one. The questions were whether the student could identify a menstrual hygiene material and whether any of the following was true: menstruation is controlled by hormones, older women experience menopause, after menarche an adolescent who engages in unprotected sex can become pregnant, menstrual blood comes from the uterus, menstruation is a normal biological process, menstruation is a sign of puberty and whether normal menstruation occur monthly. Adolescent girls who scored five out of the total of eight questions were deemed to have "adequate" knowledge but, those who scored below five points were described as having "inadequate" knowledge of MHM. Similarly, five questions were posed to determine their attitude towards MHM, each correct answer carried one point. Those who scored at least three points were described as having a "positive" attitude towards menstruation but those who scored below three points were described as having an "undesirable" attitude towards MHM. The questions on attitude were whether she ever missed school due to menstruation, abstained from household work due to menstruation, there are foods that menstruating girls should avoid, menstruating girls are dirty/unclean and whether menstruation is too shameful to discuss. Finally, 
five questions were used to assess the kind of practice of MHM. The questions were whether disposable pad/panty liner was commonly/always used to manage menses, pads were changed at least twice daily during bleeding, genitals were washed at least once daily during menstruation, hands were washed before and after changing menstrual hygiene materials and whether they were able to change pads in school whenever necessary. Those who scored at least three out of five were described as practising "good" MHM whereas those who scored less than three were described as practicing "poor" MHM. Every correct answer was coded as one but, incorrect answers coded as zero. Logistic regression was used to uncover the predictors of knowledge, attitude and practice of MHM. Only factors with statistically significant associations are reported here.

\section{Results}

\section{Socio-demographic characteristics of participants}

In all, 412 adolescent female students participated. The ages of participants ranged between 10 and 19 years with a mean of $14.4 \pm 2.1$ years. Table 1 shows that there was almost an equal number of younger (10-14 years) and older (15-19 years) adolescent girls (49.3\% against 50.7\%) respectively. There were more students in the first-year class 148 (35.9\%) than JHS two and JHS three, (33.5\% and 30.6\%) respectively. Most adolescent girls $235(57 \%)$ lived with both parents whiles $16.3 \%$ lived with either single parent.

Table 1: Socio-demographic characteristics of participants

\begin{tabular}{|c|c|c|}
\hline Age & $\begin{array}{l}\text { Number of adolescent } \\
\text { girls }\end{array}$ & Percentage \\
\hline $10-14$ & 203 & 49.3 \\
\hline $15-19$ & 209 & 50.7 \\
\hline Total & 412 & 100 \\
\hline \multicolumn{3}{|l|}{ Class } \\
\hline JHS 1 & 148 & 35.9 \\
\hline JHS 2 & 138 & 33.5 \\
\hline JHS 3 & 126 & 30.6 \\
\hline Total & 412 & 100 \\
\hline \multicolumn{3}{|l|}{ Living Parent } \\
\hline Both father and mother & 235 & 57.0 \\
\hline Only father & 67 & 16.3 \\
\hline Only mother & 67 & 16.3 \\
\hline Other & 43 & 10.4 \\
\hline Total & 412 & 100 \\
\hline \multicolumn{3}{|l|}{ Menstrual status } \\
\hline Post-menarcheal adolescents & 386 & 93.7 \\
\hline Pre-menarcheal adolescents & 26 & 6.3 \\
\hline Total & 412 & 100 \\
\hline
\end{tabular}




\section{Knowledge of Menstrual Health Management}

As shown in Table 2 below, every female adolescent student in the Municipality is aware of MHM. The first source of information on MHM for over three-quarters $321(77.9 \%)$ of them is their mother. Majority of them $(91 \%)$ also receive information on MHM in school. The commonest menstrual hygiene material known to the adolescents are the disposable menstrual pad and the cloth (80.7\% each) and tampon (22\%). Although less than half (49\%) of them agree that menstruation is controlled by hormones, $65.8 \%$ confirm that older women experience menopause. About six-in-ten know that after menarche, they can become pregnant if they engage in unprotected sex. A little over half $(52.7 \%)$ know that menstrual blood comes from the uterus and $73.3 \%$ know that menstruation is a normal biological process. Most of them $(69.4 \%$ and $78.6 \%$ ) rightly agree that menstruation is a sign of puberty and that normal menstruation occurs monthly respectively. In all, 278 (67.5\%) girls scored at least five points out of a maximum of eight points.

Table 2: Knowledge of Menstrual Health Management

\begin{tabular}{lcc}
\hline Variable & $\begin{array}{c}\text { No. of adolescent } \\
\text { N=412 }\end{array}$ & $\begin{array}{l}\text { Percentage } \\
(\mathbf{1 0 0 \%})\end{array}$ \\
\hline Ever heard of MHM (awareness) & & \\
Yes & 412 & 100 \\
No & 0 & 0 \\
Total & 412 & 100 \\
First Source of information on MHM & & \\
Mother & 321 & 77.9 \\
Sister/friend & 50 & 12.1 \\
Teacher & 23 & 5.6 \\
Others & 18 & 4.4 \\
Total & 412 & 100 \\
Ever received menstrual information in a course in school & \\
Yes & 375 & 91 \\
No & 37 & 9 \\
Total & 412 & 100 \\
Knowledge of menstrual hygiene materials (Multiple answers) & \\
Disposable sanitary pad & 305 & 80.7 \\
Reusable cloth & 305 & 80.7 \\
Tampon & 83 & 22 \\
Menstrual cup & 28 & 7.4 \\
Menstruation is controlled by hormones & 202 & \\
True & 210 & 49 \\
False & 412 & 51 \\
Total & 251 & 100 \\
Older women eventually stop menstruating & 65.8 \\
True & 271 & 34.2 \\
False & 141 & 100 \\
Total & 412 & 60.9 \\
After menarche, you can become pregnant if you have unprotected sex \\
True & 251 & \\
& & \\
& &
\end{tabular}




\begin{tabular}{lcc} 
False & 161 & 39.1 \\
Total & 412 & 100 \\
Menstrual blood comes from the uterus & & \\
True & 217 & 52.7 \\
False & 195 & 47.3 \\
Total & 412 & 100 \\
Menstruation is a normal biological process & \\
True & 302 & 73.3 \\
False & 110 & 26.7 \\
Total & 412 & 100 \\
Menstruation is a sign of puberty & & \\
True & 286 & 69.4 \\
False & 126 & 30.6 \\
Total & 412 & 100 \\
Menstruation occur every month & & \\
True & 324 & 78.6 \\
false & 88 & 21.4 \\
Total & 412 & 100 \\
Knowledge of MHM & & \\
Adequate (scored at least 5 points) & 278 & 67.5 \\
Inadequate (scored below < 5 points) & 134 & 32.5 \\
Total & 412 & 100 \\
\hline
\end{tabular}

\section{The attitude of adolescent female students towards Menstruation}

As shown in Table 3 below, almost 9-in-10 students ever missed school due to menstruation. The main reasons for missing school include fear of odour/staining or being teased 256 (76.4\%). Pain, lack of water/convenient place to wash, lack of disposal facility, unclean/no latrine to change and no access to pads/cloths in school $(77.9 \%, 64.2 \%, 59.7 \%, 74.3 \%$ and $86.9 \%$ respectively) are the main reasons for missing school. Almost two-thirds 246 $(63.9 \%)$ of post-menarcheal adolescents abstain from doing some household work due to menstruation. Two hundred and eighty-one of them $(68.2 \%)$ believe that menstruating girls should avoid some food and another $70.1 \%$ think that menstruating girls are dirty/unclean. Majority 237 (57.5\%) of them say menstruation is too shameful to discuss. Overall, $13.6 \%$ of them had three or more positive attitude answers out of five questions; the rest gave less than three positive attitude answers.

Table 3: Attitude of adolescent female students towards Menstruation

\begin{tabular}{lll}
\hline Variable & $\begin{array}{l}\text { No. of adolescent } \\
\text { girls }\end{array}$ & Percent \\
\hline Ever missed school because of menstruation & 331 & 85.8 \\
Yes & 55 & 14.2 \\
No & 386 & 100 \\
Total & & \\
Main reasons for missing school (Multiple answers) & 76.4 \\
$\begin{array}{l}\text { Afraid of odour/being teased/ staining } \\
\text { clothes }\end{array}$ & 256 &
\end{tabular}




\begin{tabular}{lcc} 
Pain & 261 & 77.9 \\
Lack of water/place convenient place to wash & 215 & 64.2 \\
Unclean/no latrine to change pad & 249 & 74.3 \\
No access to pads or cloths in school & 291 & 86.9 \\
Abstained from household work during & menstruation & \\
Yes & 246 & 63.9 \\
No & 139 & 36.1 \\
Total & 385 & 100 \\
There are some food girls should avoid during menstruation & \\
True & 281 & 68.2 \\
False & 131 & 31.8 \\
Total & 412 & 100 \\
Girl is dirty or unclean during menstruation & \\
True & 289 & 70.1 \\
False & 123 & 29.9 \\
Total & 412 & 100 \\
It is too shameful to discuss menstruation & & \\
Yes & 237 & 57.5 \\
No & 175 & 42.5 \\
Total & 412 & 100 \\
Attitude towards menstruation & & \\
Positive & 56 & 13.6 \\
Undesirable & 356 & 86.4 \\
Total & 412 & 100 \\
\hline
\end{tabular}

\section{Practices of Menstruation Health Management among adolescent female students}

In table 4 below, a fifth of the adolescents $82(21.2 \%)$ and $3.1 \%$ use disposable pads and panty liners respectively, to manage menstruation. The remaining majority use potentially unclean/unsafe materials such as cloth $(57 \%)$, cotton $(9.6 \%)$ and pieces of mattresses $(9.1 \%)$. Out of the $258(72.9 \%)$ adolescents who have ever been unable to buy a pad, majority (49.6\%) cite lack of money, followed by unavailability/long distance to shop (31.4\%) and fear of stigma (16.7\%). When they need to buy menstrual materials such as pad or panty-liners, parents/guardians are their main source of funds $(72.9 \%)$. During menstruation majority $213(55.2 \%)$ of the female adolescent students take their bath twice daily and about a third $(28.2 \%)$ bath more than twice daily. Additionally, most menstruating adolescents 201 (52.1\%) change their pads twice a day and a few (2.3\%) do not change in a day. Nine-in-ten adolescents $351(90.0 \%)$ wash their genitals at least once a day during menstruation. Majority $252(72 \%)$ of adolescents use soap and water whenever they needed to wash any soiled menstrual material. Two hundred and twenty-seven (58.8\%) wash their hands after handling used MHM 
materials whiles $19.7 \%$ each, either wash their hands before and after handling used MHM materials or do not wash their hands. Half $193(50 \%)$ of the girls are able to change their pads in school whenever necessary. Overall, a third $120(31.1 \%)$ of them indicated at least three correct answers on MHM practice.

Table 4: Menstrual hygiene management practices among adolescent female students

\begin{tabular}{|c|c|c|}
\hline Variable & No. of adolescent girls & Percent \\
\hline \multicolumn{3}{|c|}{ Materials commonly used to manage menses } \\
\hline Disposable sanitary pad & 82 & 21.2 \\
\hline Cloth & 220 & 57 \\
\hline Cotton & 37 & 9.6 \\
\hline Mattress & 35 & 9.1 \\
\hline Panty liner & 12 & 3.1 \\
\hline Total & 386 & 100 \\
\hline \multicolumn{3}{|c|}{ Ever been unable to buy sanitary pad } \\
\hline Yes & 258 & 72.9 \\
\hline No & 96 & 27.1 \\
\hline Total & 354 & 100 \\
\hline \multicolumn{3}{|c|}{ Reason for inability to buy sanitary pad } \\
\hline No Money & 128 & 49.6 \\
\hline Shop unavailable/far & 81 & 31.4 \\
\hline Fear of stigma & 43 & 16.7 \\
\hline Others & 6 & 2.3 \\
\hline Total & 258 & 100 \\
\hline \multicolumn{3}{|c|}{ Source of money for sanitary pads } \\
\hline Guardians & 283 & 72.9 \\
\hline Friends & 44 & 11.3 \\
\hline Teachers & 14 & 3.6 \\
\hline Self & 47 & 12.2 \\
\hline Total & 388 & 100 \\
\hline \multicolumn{3}{|c|}{ Number of baths taken during bleeding days } \\
\hline Once only & 60 & 15.5 \\
\hline Twice only & 213 & 55.2 \\
\hline Three times or more & 109 & 28.2 \\
\hline Can't remember & 4 & 1.1 \\
\hline Total & 386 & 100 \\
\hline \multicolumn{3}{|c|}{ Number of times pads are changed daily during bleeding } \\
\hline Once & $97^{\circ}$ & 25.1 \\
\hline Twice & 201 & 52.1 \\
\hline Three times or more & 79 & 20.5 \\
\hline None & 9 & 2.3 \\
\hline Total & 386 & 100 \\
\hline \multicolumn{3}{|c|}{ Washes genitals at least once a day } \\
\hline Yes & 351 & 90.9 \\
\hline No & 35 & 9.1 \\
\hline Total & 386 & 100 \\
\hline \multicolumn{3}{|c|}{ Materials used to wash menstrual materials } \\
\hline Water only & 98 & 28 \\
\hline Soap and water & 252 & 72 \\
\hline Total & 350 & 100 \\
\hline
\end{tabular}




\begin{tabular}{lcc} 
Mode of hand washing during Menstruation & \\
Before and after & 76 & 19.7 \\
Before & 7 & 1.8 \\
After & 227 & 58.8 \\
None & 76 & 19.7 \\
Total & 386 & 100 \\
Are you able to change pad in school whenever necessary & \\
Yes & 193 & 50 \\
No & 193 & 50 \\
Total & 386 & 100 \\
MHM Practice & & \\
Good & 120 & 31.1 \\
Poor & 266 & 68.9 \\
Total & 386 & 100 \\
\hline
\end{tabular}

Factors associated with knowledge, attitude and MHM practices

The findings in table 5 below show a statistically significant association between older adolescent female students and adequate knowledge of MHM (OR 2.616 [95\% CI, 1.708, 3.526]; P<0.001). Additionally, older adolescent female students are two times more likely to practice proper MHM compared to younger adolescent female students (OR 2.030 [95\% CI, 1.296, 3.182]; $\mathrm{P}=0.002)$. The association between those who stay with both parents and having adequate knowledge of MHM is relatively strong (OR 12.3 [95\% CI, 4.143, 29.578]; $\mathrm{P}<0.001$ ). Moreover, female adolescent girls who live with their mothers only are almost 8 times more likely to practice appropriate MHM compared to those staying alone or with other relatives (OR 7.861 (95\% CI, $2.481,24.904) ; \mathrm{P}<0.001)$. There is also a marginal association between those who have adequate knowledge of MHM and practice of MHM (OR 1.639 [95\% CI, 1.004, 2.674]; $\mathrm{P}=0.048$ ). Additionally, there is a statistically significant relationship between access to funds and good practice of MHM (OR 12.186 [95\% CI, 5.016, 29.604]; $\mathrm{P}=0.013$ ). Moreover, a statistically significant association exists between those who are given an amount of $\mathrm{Gh} \phi 2$ or more and practising good MHM. Specifically, there is a statistically significant relationship between those who receive $\mathrm{Gh} \phi 2-\mathrm{Gh} \phi 4$ and good pr actice of MHM (OR 2.369 [95\% CI 1.005, 5.585]; $\mathrm{P}=0.049)$; Gh $\varnothing 5$ Gh $\phi 6$ and good practice of MHM (OR 2.933 [95\% CI 1.461, 5.890]; P = 0.002 ); and those who are given more than Gh $\notin 6$ and good practice of MHM (OR 6.154 [95\% CI, 1.462, 25.901]; $\mathrm{P}=0.013$ ).

Table 5. Determinants, good practices and adequate knowledge of MHM

\begin{tabular}{|c|c|c|c|c|}
\hline \multirow[t]{2}{*}{ Variable } & \multicolumn{2}{|c|}{ Good MHM practice } & \multicolumn{2}{|c|}{ Adequate knowledge } \\
\hline & Odds Ratio & P-value & Odds Ratio & P-value \\
\hline & & & & \\
\hline $\begin{array}{l}\text { Older adolescents (15-19 } \\
\text { years) }\end{array}$ & $\begin{array}{l}2.030 \\
3.182)\end{array}$ & 0.002 & $\begin{array}{l}2.616 \quad(1.708, \\
3.526)\end{array}$ & $<0.001$ \\
\hline
\end{tabular}




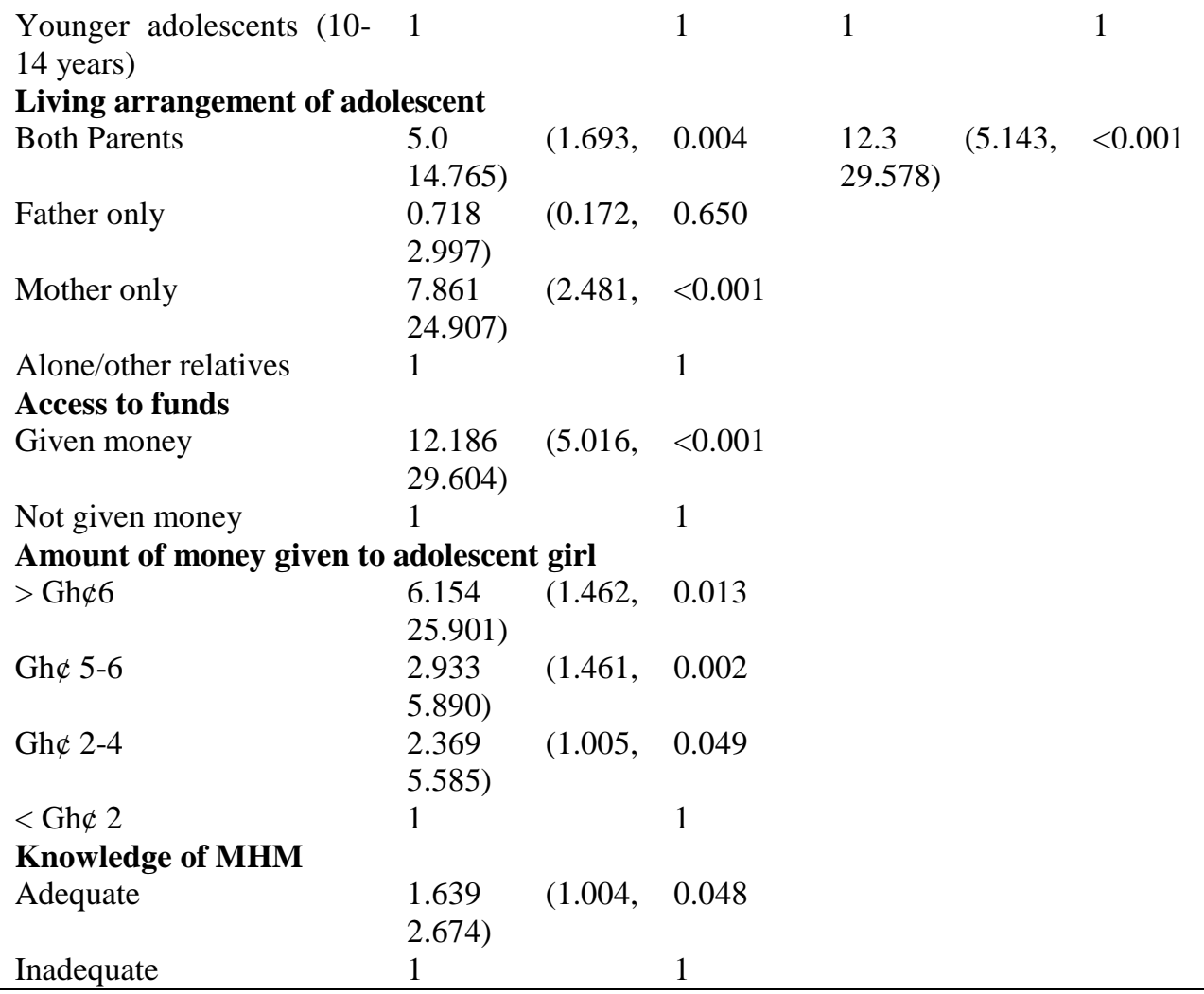

\section{Discussions}

This study's findings show that mothers were the adolescent female students' first main source of information on MHM (78\%). This seems to reinforce the important role of mothers in adolescent MHM education as suggested by (Upashe et al., 2015). The finding is in line with Chandra-Mouli and Patel [1] conclusion that mothers are the main source of MHM information for most adolescent girls in developing countries. It is however in dissonance with (Yadav et al., 2017) finding that teachers are the main source of MHM information for female adolescent students. The implication of this finding is that it is unlikely that all those who received MHM information from their mothers had accurate information because some mothers may not be well-informed about MHM. This suggests that interventions aimed at improving knowledge of MHM among adolescents in the Yendi Municipality should include mothers. Although this study revealed very few of them had their first information on menstrual hygiene from teachers, $90 \%$ of them ever received MHM information from the school at some point in time. This suggests that most of them heard about MHM from their mothers or elsewhere prior to being taught in school. This finding shows that teachers are the perhaps the second most important source of MHM information for the 
majority of them. (Yadav et al., 2017) made similar findings in their study among adolescent students in Nepal. When asked to mention the menstrual materials they knew, 80\% each, mentioned disposable sanitary pads and reusable cloths. This revelation is similar to findings by (Barthakur \& Barkataki, 2017) and (Van Eijk et al., 2016). Most disposable sanitary pads are locally produced, marketed through the mass media and sold in virtually every market. That may explain the high level of knowledge of the disposable pad among them. A fifth of them mentioned tampons and menstrual cups although none of them ever used those materials, which suggests that they obtain the information the literature or other media source. Contrary to (Okafor-Terver \& Chuemchit, 2017) findings of a low level of knowledge among female adolescent student in Nigeria, this study discovered that majority $(73.3 \%)$ of them knew that menstruation is a normal biological process. Moreover, a majority (78.6\%) of them knew that the menstrual cycle is monthly and that older women experience menopause (65.8\%). However, in consonance with (Tegegne \& Sisay, 2014) this study found that less than half of the girls knew that hormones regulated the menstrual cycle. The inconsistency in their knowledge of menstruation is further highlighted by the fact that 3 in 5 adolescent girls knew that after menarche, they are at risk of pregnancy if they engage in unprotected sex. This means that, although a high number of adolescents were aware of MHM, much of their knowledge was either inaccurate or incomplete. This calls for the intensification of reproductive health education in basic schools in Yendi. However, since most socio-cultural norms prohibit explicit discussion of sexual and reproductive health issues among adolescents, the Ministry of Education (MOE) and the Ghana Education Service (GES) must supervise and enforce the implementation in schools. Contrary to the lower level of knowledge among adolescents in Uganda, Nepal, Ethiopia and Nigeria (Yadav et al., 2017; Okafor-Terver, 2017; Ghimire, 2017) overall, 67.5\% of female adolescent students in Yendi Municipal had adequate knowledge of MHM. The discovery compares favourably with the findings of (Upashe et al., 2015) but, lower than the $90 \%$ found among adolescents in Ethiopia by (Gultie et al., 2014). The disparities may be due to the differences in scoring the Likert scales, sociocultural norms and the effectiveness of the educational system. Unlike (Gultie et al., 2014) this study found that the determinants of adequate knowledge of MHM were being an older adolescent and staying with both parents $(\mathrm{P}<0.05)$. This might be due to the fact that older adolescent girls may have received MHM education several times. Older adolescents are also more likely to have obtained menarche thus, started to experience MHM personally. Similarly, staying with both parents could mean the adolescent has more than one source of obtaining MHM information. 
Similarly, despite the relatively high level of knowledge among the girls, about a tenth $(13.6 \%)$ of them had a positive attitude towards MHM. About 9-in-10 adolescent girls ever missed school at least once, due to menstruation, which is higher than the $24 \%$ found by (van Eijk, et al., 2016) among adolescent female students in India. Lack of access to pads in school (89.6\%) and lack of functional latrines in school (74.3\%) were the two main reasons adolescent girls missed school during menstruation. This suggests that menstruation could negatively affect the academic performance of female adolescent students. Moreover, their inability to practice proper MHM in school puts them at risk of infection such as dysplasia and cervical cancer. In 2010, the Government's policy was that all basic schools in Ghana must have functional WASH facilities by 2015 (GOG, 2012). Government and UNICEF have made efforts to improve the availability of WASH facilities in basic schools, including the provision of water to schools and free sanitary pads to schooling girls. However, this study's findings suggest that their efforts have been inadequate as lack of WASH facilities remains a major barrier to school attendance by adolescent girls. Stronger political will is needed to implement school WASH policy as it requires huge capital investment. In some of the schools where UNICEF helped establish WASH facilities, most of them may have become dysfunctional due to inadequate financial support and oversight. The participation of the community members especially parents could help in sustaining WASH facilities in schools. Additionally, this study found that more than three-fifths of the adolescent girls abstained from doing household chores during menstruation. Although this attitude suggests poor knowledge of menstruation, the influence of their socio-cultural environment is apparent. For example, there are known local taboos that forbid menstruating adolescents from preparing certain foods such as "wasawsa" (a local dish made from yam). Since cooking is predominantly the role of the female gender, adolescent girls who are menstruating are unable to cook it and may not have access to the food during that period. This could have a negative impact on the adolescent girl's nutrition if wasawasa is the only food available to the family. Others erroneously believed that certain foods that contain sweets including honey could cause menorrhoea. This misconception has been reported by over 2 -in-3 adolescent student girls. Additionally, the perception that adolescent girls are dirty as indicated by $70.1 \%$ might also explain why some adolescents abstain or are prevented from carrying out their domestic responsibilities. This implies that further education on menstruation is necessary for both the adolescent girls and the communities in which they live. Moreover, the perception of $57.5 \%$ of them that menstruation is too shameful for open discussion influences the manner in which they acquire MHM information. This partly explains the practice of menstrual hygiene in silence and secrecy. This implies that educational session must assure adolescents that 
there is nothing shameful in menstruation, which is a normal natural biological process. Such assurance could enable them to contribute effectively to MHM discussions and adopt a more positive attitude towards menstruation. The study found no statistically significant factors associated with a positive attitude towards MHM. This suggests that the factors that influence an attitudinal change of female adolescent students in Yendi Municipal may be outside the scope of factors covered by this study.

Moreover, the study found that the commonest menstrual material used by the adolescent girls is cloth $(57 \%)$. This is not in consonance with the 26.9\% uncovered by (Okafor-Terver \& Chuemchit, 2017) among adolescent female students in Nigeria. However, (Upashe et al., 2015) and (Tegegne, 2014) made similar findings that majority of adolescent female students in Ethiopia use pieces of cloth to manage menstruation. In addition, (ChandraMouli \& Patel, 2017) and (Kuhlmann et al., 2017) came to the same conclusion that most adolescent girls in developing countries use a cloth to manage menstruation. Improved reusable pads are commercially available, but they are not commonly available in Yendi Municipality and most adolescents cannot afford the available ones. Since old cloth/rags are sometimes used by the adolescents, they may not be hygienic enough to prevent infection. As reported by (Trinies et al., 2015) in neighbouring Mali some of them dry the washed pieces of MHM cloths indoors to avoid a spiritual attack on their fertility, which increases the risk of infection. This also is an indication that local socio-cultural beliefs may be having a negative influence on the knowledge, attitude and practices of MHM by adolescent female students Yendi Municipal. A qualitative research would be necessary to unearth the nuances around beliefs and MHM practices among adolescents in Yendi Municipal. It is worth noting that adolescents who are unable to secure cotton cloth materials may experience poor absorption. Moreover, when the user is engaged in vigorous physical exercise such as playing football or experiencing menorrhoea the rug may fail to provide proper protection. This may partly explain why some adolescent girls think that it is bad for menstruating girls to engage in physical exercise, as physical activity increases the risk of the cloth shifting and causing leakage.

Majority of girls (72.9\%) could not buy disposal menstrual pads mainly due to lack of funds. This resonates with findings among adolescent female students in rural Kenya by (McMahon et al., 2014). This situation is partly due to the fact that majority of them are below the legal age of employment, thus do not have an independent income. Yendi Municipality is among the most gendered communities where unpaid domestic labour such as cooking, fetching water and cleaning are strictly the responsibility of adolescent girls and women. Their lack of independent income reinforces their dependence on parents and male friends for funds to buy pads. That 
notwithstanding, 1-in-10 adolescents were able to buy pads from their personal savings. What is worrying is the discovery that more than a tenth $(16.7 \%)$ of the adolescent girls could not purchase a sanitary pad for fear of stigma. Issues of menstruation are often conducted clandestinely and the adolescent girls said they could not purchase a pad when their male counterparts were nearby for fear of stigma. Similar findings were made by (McMahon et al., 2014) among adolescent female students in rural Kenya where stigma was a major cause of psychosocial problems associated with menstruation. Whether the threat of stigma among the adolescent student girls in Yendi Municipal was perceived or actual, the findings suggest a significant number of the adolescent girls need life skills training to enable them to acquire pads whenever they need them. Similarly, there is the need to sensitise male students and teachers and the community to support rather than ridiculing menstruating girls.

In terms of menstrual hygiene practice, the study also found that nearly all $(98.9 \%)$ adolescent girls bath at least once during menstruation. More than half $(55.2 \%)$ of them bath twice a day at home during menstruation. This is lower than the $67.3 \%$ among their counterparts in Ethiopia (Upashe et al., 2015). This suggests that there are no social restrictions on bathing for menstruating adolescents girls in their period. The practice of regular bathing is perhaps possible because it is a norm for students in Ghana to bath before going to school. This must, however, be complemented by the provision of WASH facilities in schools to enable girls to clean themselves as often as necessary. Moreover, contrary to (van Eijk, et al., 2016) findings that a third of adolescent females in India changed their pads in schools, at least half $(52.1 \%)$ of those in Yendi changed their pads twice daily. This is necessary as it reduces the likelihood of the pad leaking from soaking beyond capacity. It is also healthy as it reduces the risk of acquiring reproductive tract infections (RTI) and toxic shock syndrome (TSS) from prolonged exposure to potentially infectious blood. A few (2.3\%) used the same unchanged pad for a day, which might be due to lack of access to pads. Although a majority $(90.1 \%)$ of adolescent girls wash their genitals at least once a day, most of them $(58.8 \%)$ do so only after changing pads, or handling used menstrual materials. Similar attachment of importance to the practice of hand washing has been recorded among 92.2\% adolescent female students in Nigeria (Okafor-Terver \& Chuemchit, 2017). About 1-in-5 would not wash their hands after handling used menstrual products or changing pads. The lack of water (especially in schools) could be the main reason for not washing their hands after handling used menstrual materials. The girls reported burying, burning or wrapping and putting used menstrual material into refuse bins, all of which are safe disposal methods. Since most schools' lack disposal facilities, most menstruating adolescent students will be unable to change their absorbents and may absent 
themselves from school until the flow is over. Overall, a third of the girls practised appropriate menstrual hygiene management. This means that despite the high knowledge, several factors such as lack of funds to buy pads, lack of WASH and disposal facilities in schools are barriers to practising appropriate menstrual hygiene.

The study also revealed that having adequate knowledge of MHM, having access to funds and achieving the age of older adolescence (15-19 years) were important predictors of good MHM practice $(\mathrm{P}<0.05)$. This means that more education on MHM could lead to the increased practice of proper MHM although not necessarily attitudinal change. For effective MHM behaviour modification among adolescent girls, parents and boys should be included in MHM education to reduce the stigma associated with acquiring absorbents. Similarly, those staying with both parents are not only more likely to receive funds to buy materials, but also likely to be coached on menstrual hygiene compared to those not staying with any parent. Mothers may be more comfortable talking MHM with their daughters than fathers, which may explain their link to the adolescent knowledge of MHM. Moreover, this study's findings that access to funds, irrespective of the amount was a predictor of proper MHM practice, suggest that poverty is a contributory factor in not practising proper menstrual hygiene. It also implies that the financial cost of pads may is a barrier for poor adolescent female students. Girls who had access to funds may have come from families of higher socio-economic status and could afford to purchase and use clean sanitary pads. The link between access to funds and MHM practice is not new. One study in Kenya revealed that addition to stigma, menstruation can be impoverishing to adolescent girls as some of them have to borrow money to buy pads or feed during menstruation (Sommer et al., 2015). Attempts to distribute free pads to students in Ghana have proved to be unsustainable. The current finding suggests that Government make are more sustainable policy such as removing taxes on MHM products to make them more affordable to adolescent girls. MHM products can be considered as merit goods, thus the removal of taxes and the accompanying cost reduction could lead to improved consumption among student adolescent girls. Moreover, such an intervention could lead to improved gender equity as it removes the unfair impoverishing financial burden that goes with managing menstrual hygiene. India for instance, has scrapped taxes on menstrual products (sometimes called blood tax) (BBC, July 2018). Finally, the relationship between age and MHM practice may be due to the fact that older adolescent student girls may be able to work and/or have older friends with income who can support them financially to acquire and use more hygienic menstrual materials. 


\section{Conclusion}

Although a majority of adolescent girls had knowledge of menstruation, less than a third had a positive attitude towards menstruation or practised appropriate menstrual hygiene management. This has been partly attributed to the negative socio-cultural norms and practices that stigmatise menstruation, lack of MHM materials and WASH facilities in schools, inadequate access to funds to purchase clean pads, and ignorance of menstrual hygiene management. In a nutshell, the findings suggest that majority of female adolescent students in Yendi Municipality have an unmet need for menstrual hygiene management. A qualitative study is necessary to explore the socio-cultural factors influencing adolescent students' attitude and practice of menstrual hygiene management.

\section{Recommendations}

To the GES and MOE, the study recommends that they implement fully, the WASH policy in basic schools in the Yendi Municipality. This may be achieved by involving the communities in the provision and management of WASH facilities in schools to ensure sustainability.

Secondly, that they collaborate with the Ghana Health Service to train both parents and teachers on proper menstrual hygiene management. This can be done through the involvement of parents and teachers associations in the various schools.

Thirdly, that they collaborate with the National Commission for Civic Education to sensitise the communities to change the negative socio-cultural norms and practices that stigmatise menstruation.

Additionally, that they collaborate with researchers to further explore the factors that hinder menstrual hygiene practice among female adolescent students in the Municipality.

Finally, to the Government of Ghana, the study recommends that it remove taxes on menstrual hygiene management products to make them more affordable for adolescent girls in basic schools. This will not only sustainably help improve the consumption of safe MHM products but improve gender equity.

\section{References:}

1. Alam M, U, Luby S, P., Halder A, K., Islam K, Opel A, Shoab A, K., et al., (2017); 7: e015508. Menstrual hygiene management among Bangladeshi adolescent schoolgirls and risk factors affecting school absence: results from a crosssectional survey. BMJ Open.

2. Barthakur C, Barkataki M., (2017); 8 (10): 602-5. A Study on Reproductive Health Problems and Menstrual Hygiene Practices 
among Adolescent Girls Living in Slums of Guwahati city, Assam. Natl J Community Med.

3. BBC, (July 21 2018). India scraps tampon tax after campaign [press release].

4. Boosey R, Prestwich G, Deave T., (2014); 19: 253. Menstrual hygiene management amongst schoolgirls in the Rukungiri district of Uganda and the impact on their education: a cross-sectional study. Pan Afr Med J..

5. Chandra-Mouli V, Patel S, V., (2017); 14(1):30. Mapping the knowledge and understanding of menarche, menstrual hygiene and menstrual health among adolescent girls in low- and middle-income countries. Reproductive Health.

6. Dutta D, Badloe C, Lee H, House S., (2016). Supporting the Rights of Girls and Women through Menstrual Hygiene Management (MHM) in the East Asia and Pacific Region: Realities, Progress and Opportunities. Bangkok, Thailand: UNICEF East Asia and Pacific Regional Office (EAPRO).

7. Ghana Statistical Service, (2010). Population and Housing Census. District Analytical Report, Yendi Municipality. Accra, Ghana: GSS, 2014.

8. Ghimire S., (2017); 5 (8): 3426-30 Knowledge regarding menstrual hygiene among adolescent girls. Int J Res Med Sci..

9. Government of Ghanan, (2012). Education Strategic Plan 2010-2020: Policies, Strategies, Delivery, Finance. Accra, Ghana: GES.

10. Gultie T, Hailu D, Workineh Y., (2014); 9 (9): e108644. Age of Menarche and Knowledge about Menstrual Hygiene Management among Adolescent School Girls in Amhara Province, Ethiopia: Implication to Health Care Workers \& School Teachers. PLoS ONE.

11. Hennegan J, Dolan C, Wu M, Scott L, Montgomery P., (2016); 13: 143. School girls' experience and appraisal of menstrual absorbents in rural Uganda: a cross-sectional evaluation of reusable sanitary pads. Reproductive Health.

12. Hennegan J, Montgomery P., (2016); 11 (2): e0146985. Do Menstrual Hygiene Management Interventions Improve Education and Psychosocial Outcomes for Women and Girls in Low and Middle Income Countries? A Systematic Review. PLoS ONE.

13. Ibitoye M, Choi C, Tai H, Lee G, Sommer M., (2017); 12 (6): e0178884. Early menarche: A systematic review of its effect on sexual and reproductive health in low- and middle-income countries. PLoS ONE. 
14. Kuhlmann A, S., Henry K, Wall L, L., (2017); 72 (6): 356-76. Menstrual Hygiene Management in Resource-Poor Countries. Obstetrical \& gynecological survey.

15. McMahon S, A., George A, S., Chebet J, J., Mosha I, H., Mpembeni R, N., Winch P, J., (2014); 14 (1): 1. Experiences of and responses to disrespectful maternity care and abuse during childbirth; a qualitative study with women and men in Morogoro Region, Tanzania. BMC pregnancy and childbirth.

16. Miiro G, Rutakumwa R, Nakiyingi-Miiro J, Nakuya K, Musoke S, Namakula J, et al., (2018); 18: 4. Menstrual health and school absenteeism among adolescent girls in Uganda (MENISCUS): a feasibility study. BMC Women's Health.

17. Montgomery P, Ryus C, R., Dolan C, S., Dopson S, Scott L, M., (2012); 7 (10): e48274 Sanitary Pad Interventions for Girls' Education in Ghana: A Pilot Study. PloS ONE..

18. Nemade D, Anjenaya S, Gujar R., (2009); 32 (4): 167-75. Impact of health education on knowledge and practices about menstruation among adolescent school girls of Kalamboli, Navi-Mumbai. Health and Population: Perspectives and Issues.

19. Okafor-Terver I, S., Chuemchit M., (2017); 31 (Suppl. 2): S179-87. Knowledge, belief and practice of menstrual hygiene management among in-school adolescents in Katsina state, Nigeria. J Health Res.

20. Santra S., (2017); 4 (3): 708-12. Assessment of knowledge regarding menstruation and practices related to maintenance of menstrual hygiene among the women of reproductive age group in a slum of Kolkata, West Bengal, India. International Journal of Community Medicine and Public Health.

21. Sommer M, Cherenack E, Blake S, Sahin M, Burgers L., (2015). WASH in Schools Empowers Girls' Education: Proceedings of the Menstrual Hygiene Management in Schools Virtual Conference 2014. Sinden J, editor. New York, USA: UNICEF and Columbia University;

22. Tegegne T, K., Sissay M, M., (2014); 14 (1): 1118. Menstrual hygiene management and school absenteeism among female adolescent students in Northeast Ethiopia. BMC Public Health.

23. Trinies V, Caruso B, A., Sogore A, Toubkiss J, Freeman M, C., (2015); 34 (1): 31-40. Uncovering the challenges to menstrual hygiene management in schools in Mali. Waterlines.

24. Upashe S, P., Tekelab T, Mekonnen J., (2015); 15 (1): 84 . Assessment of knowledge and practice of menstrual hygiene among high school girls in Western Ethiopia. BMC women's health.

25. van Eijk A, M., Sivakami M, Thakkar M, B., Bauman A, Laserson K, F., Coates S, et al., (2016): e010290. Menstrual hygiene management 
among adolescent girls in India: a systematic review and meta analysis. BMJ Open.

26. Yadav R, N., Joshi S, Poudel R, Pandeya P., (2017); 15 (37): 212-6. Knowledge, Attitude, and Practice on Menstrual Hygiene Management among School Adolescents. J Nepal Health Res Counc. 